\title{
A multiattribute decision support system with imprecise input and time-dependent modelling
}

\section{Sixto Ríos-Insua*, Antonio Jiménez and Alfonso Mateos}

Department of Artificial Intelligence, School of Computer Science, Madrid Technical University, Campus de Montegancedo s/n, 28660 Madrid, Spain

E-mail: srios@fi.upm.es E-mail: ajimenez@fi.upm.es

E-mail: amateos@fi.upm.es

*Corresponding author

\section{Tomás Prieto}

Department of Statistics and Operations Research,

Complutense University of Madrid, Plaza de las Ciencias $s / n$,

28040 Madrid, Spain

E-mail: tprieto@mat.ucm.es

\begin{abstract}
We describe a modelling and problem-solving process supported by a decision support system, which accounts for the possibility of different models depending on factors like multiple conflicting objectives, uncertainty about the consequences of the decisions, imprecision concerning the decision maker's preferences and decisions with consequences that span several time periods. This decision support system addresses all the above aspects, which can be simultaneously taken into account in real decision making problems, by considering an objective hierarchy that models appropriate time preferences. The analysis is focused on judgemental inputs, imprecise scaling factors on objectives and imprecise scalar utility functions on the attributes of the hierarchy for the purpose of aggregation, if appropriate, in a scalar or vectorial global additive utility function, which can incorporate discount factors to evaluate the timestream vectors. The decision support system includes an optimisation method based on the simulated annealing metaheuristic, as well as a multiparametric sensitivity analysis as an aid for identifying the optimal alternative.
\end{abstract}

\section{Introduction}

Expected Utility Theory (see e.g. [1-3]) has been an important approach for dealing with decision making problems under uncertainty for more than 50 years, providing a rational problem-solving framework. However, some experience with applications and the consideration of increasingly more complex real decision making problems has recently led to the provision of more robust approaches to decision making, which can account for imprecision and could overcome some of the criticisms of Decision Analysis. This has necessarily brought about the development of improved decision support systems (DSS) to deal with the increasing difficulties involved in these problems. One of the biggest obstacles is the difficulty in assessing the different model inputs for subsequent problem solving. The traditional approach required single values to be given in the utility and weight elicitation process, which is very demanding and stressful for the decision maker (DM). Recently, a more relaxed approach has been considered, which asks for ranges or intervals instead of single values. Furthermore, we consider a situation where the consequences of the possible alternatives could have uncertain effects, i.e. they could be given by means of intervals instead of single values, as suggested in [4]. 
Thus, our proposal is to develop a DSS based on a modelling approach that assumes imprecision concerning all the possible inputs considered in the model and supported by an objective tree to account for the presence of multiple conflicting objectives.

To be more realistic, we also assume the possibility of a dynamic approach in the sense of objectives spanning several time periods, which leads to the construction of an objective tree over these periods. Thus, the system will be focused on judgemental inputs and is able to capture and deal with objective trees that model appropriate imprecise time preferences by means of imprecise utility functions, taking into account the effects of discounting on the outcomes, if deemed appropriate by the DMs. This approach can aid DMs to express their opinions or judgements with a better understanding of the situation, because attributes, weights and the uncertainty surrounding them can change over time.

The DSS is based on a global additive utility function that can aggregate the different components into a scalar or vectorial utility function, depending on the inclusion of discount factors in the model to evaluate the timestream vectors and imprecision concerning the different inputs. Thus, considering all the possible combinations with respect to precision or imprecision concerning the consequences, utilities and weights, as well as the static or dynamic options, we have, in principle, the possibility of modelling 16 'pure' models, as shown in Figure 1. But it is also possible to combine precision and imprecision, as would be done in a real decision making problem, where the DMs possibly have different information levels for the parameters considered. Then, we have mixed models, and all the possibilities are supported by the DSS. 
Figure 1 Modelling tree for the different input possibilities

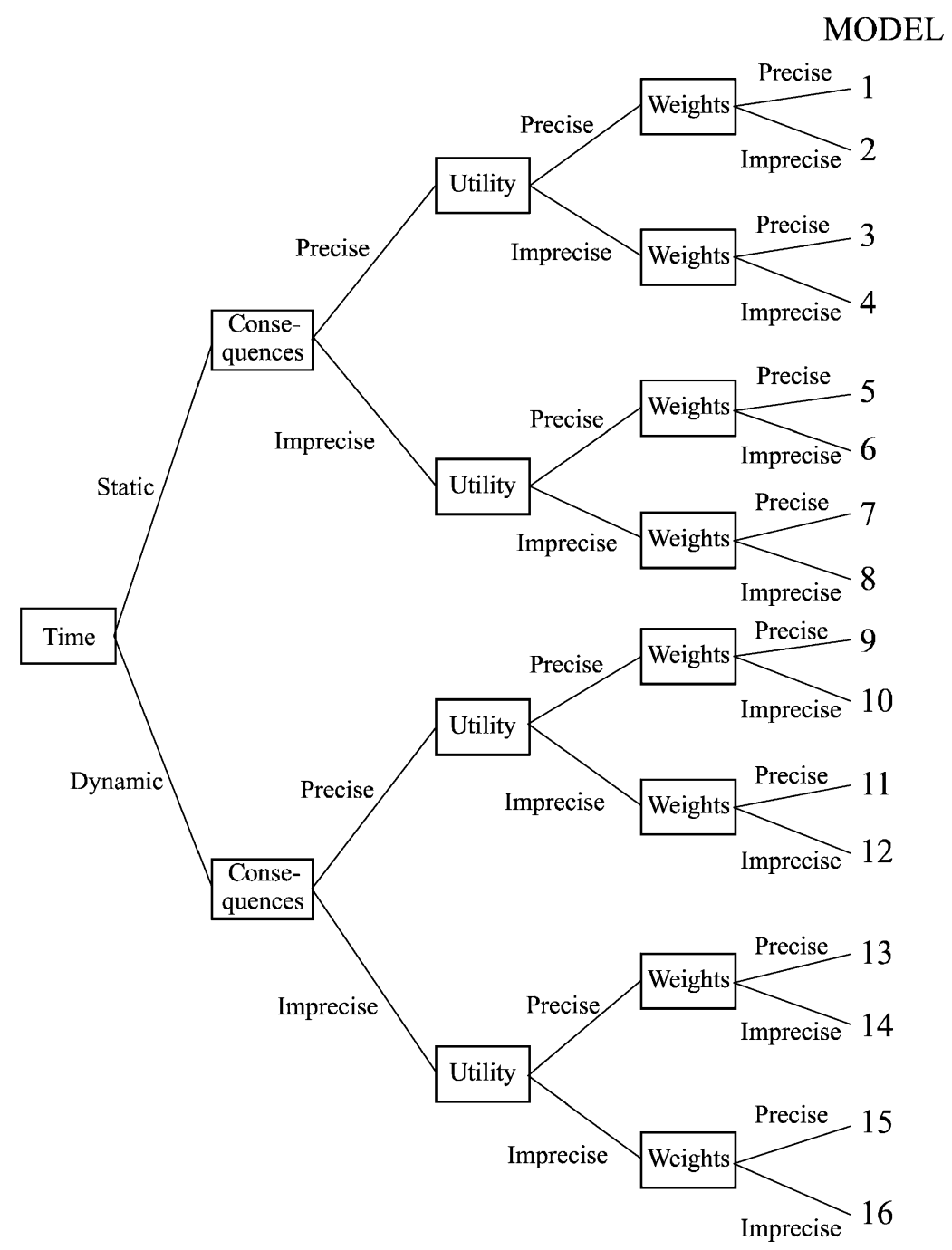

This paper describes the methodology of this evaluation system implemented in a PC-DSS, which allows for the incorporation of all relevant information to aid DMs to get the best alternative. The DSS also includes a multiparametric sensitivity analysis to check the sensitivity of the conclusions on the inputs.

The next section deals with objective tree structuring over time. Section 3 considers multiattribute utility modelling under imprecision and, Section 4, the different problem-solving approaches depending on the available information on the inputs. This includes possible discounting methods to provide DMs with assistance concerning how to trade off the present with the future and an approximation approach to determine the best alternative based on an interactive simulated annealing metaheuristic for multiple attributes. Section 5 outlines different multiparametric sensitivity analyses and, finally, a summary of the paper is given. 


\section{Objective tree structures}

There are several benefits to be gained from using a hierarchy to model decision making problems with multiple conflicting objectives, see, e.g. [5].

The objective hierarchy must include all the relevant aspects related to the problem under consideration and should be structured in such a way that objectives of similar importance are at the same level of the tree, improving the DMs' understanding of the decision.

On the other hand, attributes must be identified for the lowest-level objectives to indicate to what extent they are achieved by the alternatives under consideration. They must be specified in a similar level of detail, otherwise they will be difficult for DMs to compare. $[5,6]$ outline what properties attributes should have and discuss general structuring issues.

The DSS looks at the possibility of long-term decision making and ways of modelling the different inputs. It will be able to capture and deal with objective trees that model appropriate time preferences, i.e. which model preferences from objectives and attributes in a hierarchical structure over long time horizons by means of imprecise utility functions for measuring the DMs' preferences, taking into account the effects of different discounting methods on the decisions taken, if deemed appropriate by the DMs. Based on the above ideas, the DSS can operate with a single objective tree for the static option (see Figure 2a) and with an objective tree including time periods for the dynamic alternative (see Figure $2 b$ ).

Figure 2 Objective hierarchies for the static and dynamic options

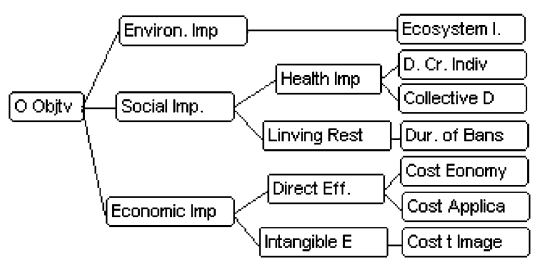

a) Static Option

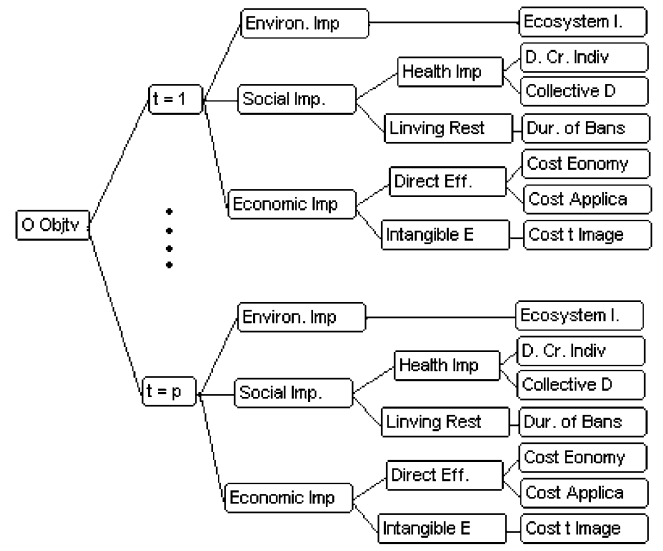

b) Dynamic Option

The objective hierarchy in the above figure represents a complex decision making problem, the restoration of aquatic ecosystems contaminated by radionuclides $([7,8])$. This problem was studied at length in several European projects in which we took part, and was the basis of the DSS we have developed. 
The attribute set for each time period could be common, but one can imagine many circumstances where they could differ. Thus, different sets of attributes describing DMs' perceptions of value in the different periods would be taken into account. Intertemporal decisions could be considered as an explicit tradeoff between the present and the future by structuring the objectives tree in such a way that the importance of the significant attributes in each period is made explicit.

As we pointed out, the DSS also allows for uncertainty about the consequences or outcomes of the alternatives under consideration ([4]) in such a way that they can be provided by means of uniformly distributed ranges or intervals instead of single values, as an approach under certainty would demand. Thus, given an $n$ attribute set denoted as $X_{1}, \ldots, X_{n}$ and $t=1, \ldots, p$ time periods (for ease we assume that the set of attributes for each time period $t$ is the same in such a way that the DMs will assign a zero weight to any fixed (given) attribute that it is not relevant in a particular time period, whose contribution will, therefore, be null), the consequence of the alternative or decision $S_{q} \in S$, where $S$ denotes the set of available alternatives to the DMs, will be an outcome stream defined by

$$
\begin{aligned}
& \left(\left(\left\lfloor x_{1 q}^{L 1}, x_{1 q}^{U 1}\right\rfloor, \ldots,\left\lfloor x_{n q}^{L 1}, x_{n q}^{U 1}\right\rfloor\right), \ldots,\left(\left\lfloor x_{1 q}^{L t}, x_{1 q}^{U U}\right\rfloor, \ldots,\left\lfloor x_{n q}^{L t}, x_{n q}^{U t}\right\rfloor\right), \ldots\right. \\
& \left.\left(\left\lfloor x_{1 q}^{L p}, x_{1 q}^{U p}\right\rfloor, \ldots,\left\lfloor x_{n q}^{L p}, x_{n q}^{U p}\right\rfloor\right)\right)
\end{aligned}
$$

where $x_{i q}^{L t}$ and $x_{i q}^{U t}$ are the lower $(L)$ and upper $(U)$ endpoints of the imprecise consequence for attribute $X_{i}$ in the time period $t$, respectively. If both endpoints were equal, i.e. $x_{i q}^{L t}=x_{i q}^{V t}$, it would be equivalent to the case under certainty, where the consequences for such alternative $S_{q}$ and attribute $X_{i}$, are precisely known. For the static option, the consequences are given only by the first set

$$
\left(\left\lfloor x_{1 q}^{L 1}, x_{1 q}^{U 1}\right\rfloor, \ldots,\left\lfloor x_{n q}^{L 1}, x_{n q}^{U 1}\right\rfloor\right) .
$$

We also consider the possibility of substituting each interval by a single value given by $x_{i q}^{A t}=\left(x_{i q}^{L t}+x_{i q}^{U t}\right) / 2$ ( $A$ means average), having then a precise consequence, if deemed appropriate by the DMs.

Now, the problem is to rank the alternatives in $S$ and, for this purpose, we provide a Decision Analysis-based evaluation procedure (see, e.g. [9-11]), with the support of an optimisation method that uses interactive multiobjective simulated annealing.

\section{Imprecise multiattribute utility function assessment}

Expected multiattribute utility theory can be considered as a leading paradigm for normative decision theory. However, multiattribute utility theory calls for the DMs to provide all the information describing the decision situation to assess the component scalar utility functions $u_{i}$ that represent the DMs' preferences concerning the possible attribute values. This could be far too strict in most practical situations, possibly leading to the consideration of imprecise component utility functions [12]. Thus, what we shall call the imprecise timestream utility efficient alternative set plays 
an important role in our modelling context, because it has a similar property to the well-known efficient or Pareto optimal alternative set for the static precise case. However, this set can be very difficult to determine in this new context and intelligent approaches are needed to help the DMs to arrive at a final solution, as we will see later.

The difficulty in assessing utility functions is well known and several authors (see, e.g. $[13,14])$ have suggested that elicited utility functions are generally method-dependent, and bias and inconsistencies may be generated in the assignment process. To overcome these problems, we have implemented a combination of two slightly modified standard procedures in the DSS: the fractile method and the extreme gambles method that belong to the certainty equivalence $(\mathrm{CE})$ and probability equivalence (PE) categories (see [15]), respectively. Moreover, it assumes imprecision in the scalar utility functions, allowing the DMs to provide a range of responses instead of only one precise value to each probability question posed, as these methods demand. This is less stressful for experts, since they are allowed to provide incomplete preference statements by means of intervals rather than single values, $[16,17]$. As a result, we get a class of utility functions for each method, rather than a single one. The intersection between the two classes provides the range where preferences elicited by the above methods agree. Should such intersections be empty for an interval, the DMs would be inconsistent and they should re-elicit their preferences. The DSS is able to detect possible inconsistencies and suggest what the DMs should change to achieve consistency. The process finishes when a consistent range is obtained.

Specifically, with the fractile method, the DMs are asked to provide the certainty equivalence intervals or attribute value intervals $\left\lfloor x_{i}^{L j}, x_{i}^{U j}\right\rfloor$ they consider equivalent to gambles, whose results are the extreme values $x_{i}^{*}$ and $x_{i^{*}}$ and probabilities $p^{j}$ and $1-p^{j}$. We take $p^{1}=0.25, p^{2}=0.50$ and $p^{3}=0.75$. This means that the DMs consider the comparisons

$$
\left(\begin{array}{cc}
p^{j} & 1-p^{j} \\
x_{i}^{*} & x_{i^{*}}
\end{array}\right) \sim x_{i}^{j}
$$

( $\sim$ means indifference) for all the amounts $x_{i}^{j} \in\left\lfloor x_{i}^{L j}, x_{i}^{U j}\right\rfloor$, for $j=1,2,3$, where $x_{i}^{*}$ and $x_{i^{*}}$ represent the most and least preferred values for attribute $X_{i}$, respectively.

The method determines a range in the $x_{i} / u_{i}\left(x_{i}\right)$-diagram, which can be represented by the bounding utility functions $u_{i}^{L_{C E}}$ and $u_{i}^{U_{C E}}$, where $L(U)$ means Lower(Upper). Figure 3 shows a possible example of this class of utility functions drawn between the soft lines. 
Figure 3 Classes of utility functions based on the CE and PE methods for attribute $X_{i}$

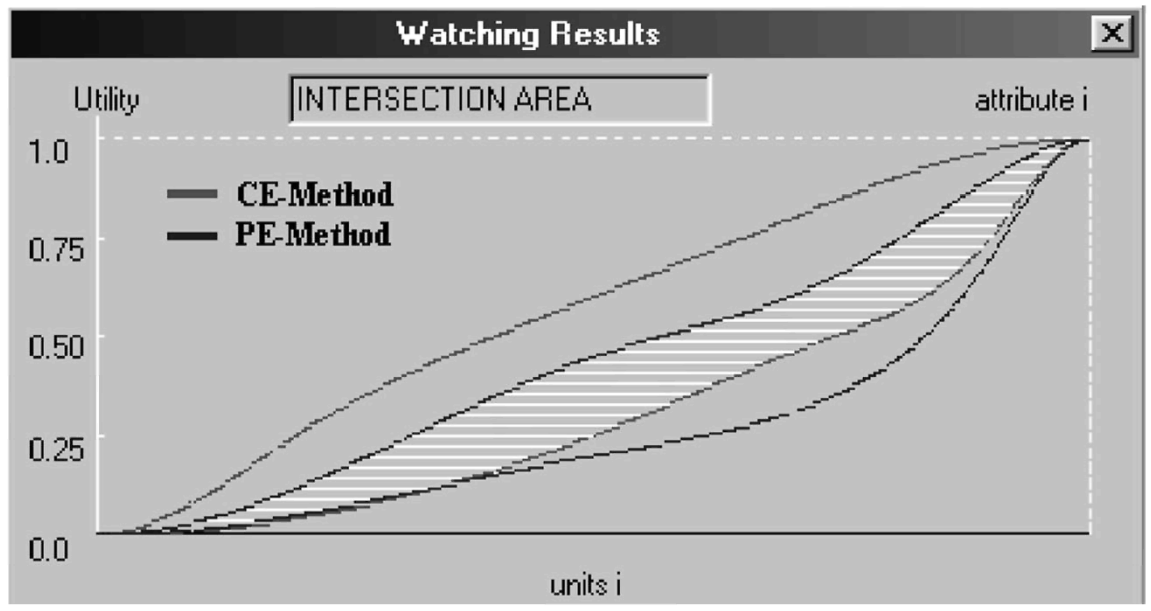

To output these probability intervals, the DSS includes a routine implementing a wheel of fortune [18], which provides the probabilistic questions and guides the expert until an interval of indifference probabilities is obtained. A number of additional questions are included as consistency checks.

With the extreme gambles method the DMs have to specify probability intervals $\left\lfloor p^{L j}, p^{U_{j}}\right\rfloor, j=1,2,3$, such that

$$
\left(\begin{array}{cc}
p^{j} & 1-p^{j} \\
x_{i}^{*} & x_{i^{+}}
\end{array}\right) \sim x_{i}^{j}
$$

for all $p^{j} \in\left\lfloor p^{L j}, p^{U_{j}}\right\rfloor$, for some selected amounts $x_{i}^{j}$ between $x_{i^{*}}$ and $x_{i}^{*}$. We use the upper bounds of the certainty equivalent intervals provided by the DMs in the $\mathrm{CE}$-method as sure amounts $x_{i}^{j}$. Other points can be used for comparison. Figure 3 also shows a possible utility function class, drawn between the black lines and represented by the bounding utility functions $u_{i}^{L_{P E}}$ and $u_{i}^{U_{P E}}$, with $L$ and $U$ as above.

Therefore, we have a utility interval $\left|u_{i}^{L}\left(x_{i}\right), u_{i}^{U}\left(x_{i}\right)\right|$ instead of a single utility $u_{i}\left(x_{i}\right)$ for each attribute $X_{i}$, which represents the elicited DMs' imprecision concerning the utility of value $x_{i}$. This is shown for the increasing utility functions in Figures 3 and 4 by the striped area. The result for decreasing utility functions would be similar.

While the lower and upper bounds of the utility function classes ( $u_{i}^{L}$ and $u_{i}^{U}$, respectively) are used to compute the lower and upper overall utility associated with each alternative, average utility functions (output by fitting natural cubic splines to the mid-points of these classes), $u_{i}^{A}$, are used to quantify their average overall utilities, which leads to the alternatives ranking, see Figure 4. 
Figure 4 Class of utility functions and precise utility function for attribute $X_{i}$

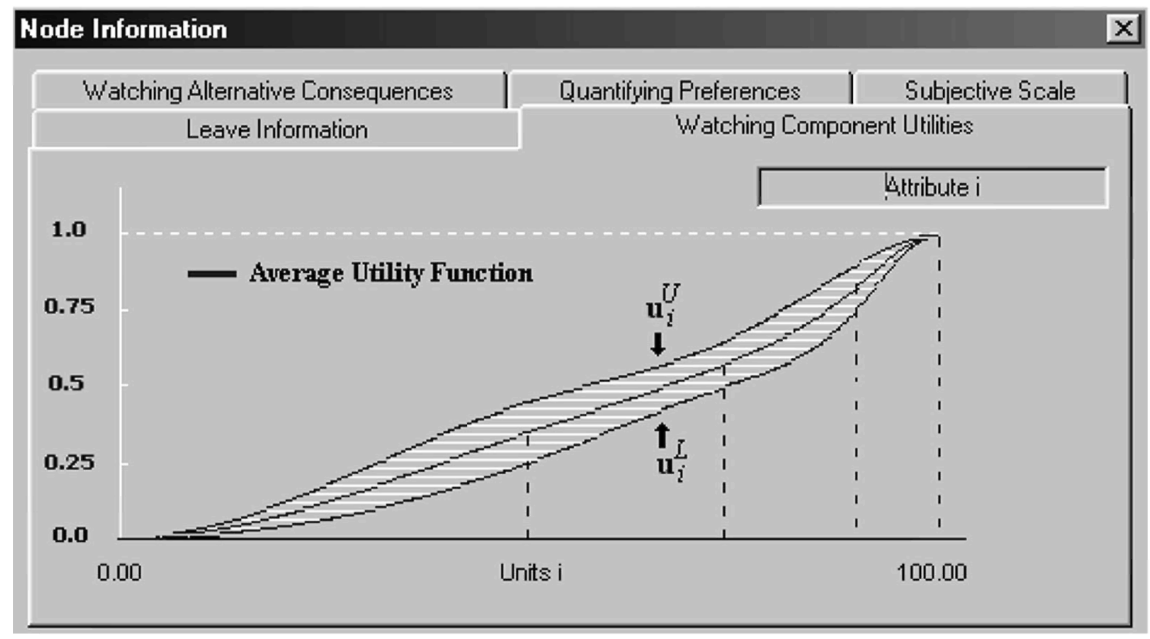

Positive scaling constants or weights $w_{i}$ for each attribute $X_{i}$ are also necessary for the additive utility function, by means of which we can add up the separate contributions of the attributes to output the total utility. As in the case of utility assessment, imprecise assignments by means of ranges or intervals are possible. This means that the DMs will be able to perform a global sensitivity analysis, allowing intervention at any level of the objective hierarchy.

Note that when the DSS is run, the starting point is equally weighted objectives. If the DMs do not agree with these objective weights, they can be reassigned using either of the two methods provided by the DSS.

The first procedure is based on tradeoffs among the respective attributes of the lowest-level objectives stemming from the same objective. The DMs are asked to provide a probability interval such that they are indifferent between a gamble and a sure consequence (see [19]). Note that the user can specify identical interval endpoints, which means that the DMs are considering a precise value. This procedure is best suited for the lowest-level objectives in the hierarchy because the weight assessment involves a more specific area of knowledge. We begin with the objectives at the lowest levels of the hierarchy and then we continue the assessment in ascending order through the hierarchy. 
Figure 5 Weight assessment based on trade-offs

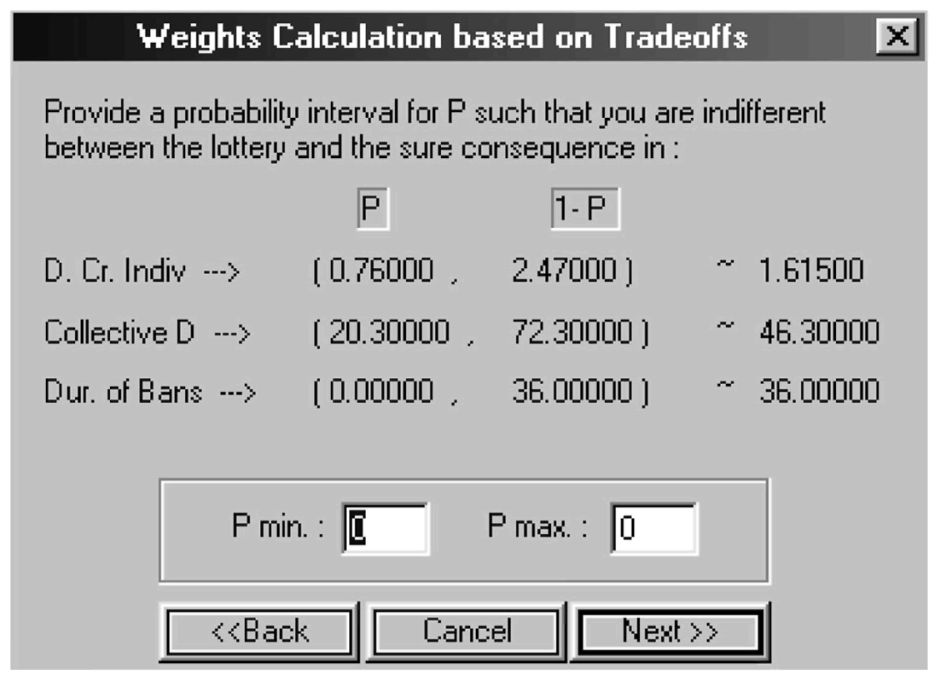

The second procedure is based on a direct assessment and is perhaps more suitable for upper level objectives, because they may be more political. Here, the DMs are asked to provide weight intervals, as before. A precise value is provided if the user specifies identical interval endpoints.

In both methods, the DSS takes the DM's imprecise responses and computes an average weight, $w_{i}^{A}$, and a normalised weight interval $\left[w_{i}^{L}, w_{i}^{U}\right]$ for each node under consideration by means of a normalisation process. As in the case of component utility functions, the DSS gets the average weights to rank the alternatives, while the normalised weight interval endpoints are used to compute lower and upper overall utilities.

\section{The underlying problem-solving processes for the possible models}

We now describe some of the possible models outlined above, which can be introduced into the DSS, as well as the associated problem-solving processes for outputting the optimal or at least a satisfactory solution for DMs. We explain three models, which represent all the possible situations that DMs could afford with the DSS, in detail. We first present model 1, which addresses the classical static case, where the inputs (utilities and weights) are precise and the consequences of the considered alternatives are certain. At the other end of the scale, we discuss, at the end of this section, model 16, which is the most complex, being time dependent and assuming imprecision in the inputs and uncertainty about the consequences, modelled by means of a continuous uniform distribution. Finally, in between these two models, we consider model 9, where the difficulty of the modelling process is medium. These detailed descriptions of the above models cover all the possibilities indicated in the modelling tree shown in Figure 1, as well as other partial combinations, because any of the remaining models have parts in common with the models described. 


\subsection{The static and precise case}

We start with the simplest case, corresponding to MODEL 1 in Figure 1. This is the classical static model with precision in all the inputs. There will be a consequence vector $\left(x_{1 q}^{A 1}, \ldots, x_{n q}^{A 1}\right)$ associated with each alternative $S_{q}$, and its average utility will be, under the additive independence condition [6] or an approximation [20,21],

$$
u\left(S_{q}\right)=\sum_{i=1}^{n} w_{i}^{A} u_{i}^{A}\left(x_{i q}^{A 1}\right) .
$$

The determination of the best alternative is a scalar optimisation problem,

$$
\begin{array}{ll}
\max & u\left(S_{q}\right) \\
\text { s.t. } & S_{q} \in S
\end{array}
$$

where the optimal alternative is the one with the highest utility.

\subsection{A dynamic case with precision. Interactive multiobjective simulated annealing}

When a precise situation as above is dynamic, we have MODEL 9, where each alternative $S_{q}$ has the associated consequence

$$
\left(\left(x_{1 q}^{A 1}, \ldots, x_{n q}^{A 1}\right), \ldots,\left(x_{1 q}^{A t}, \ldots, x_{n q}^{A t}\right), \ldots,\left(x_{1 q}^{A p}, \ldots, x_{n q}^{A p}\right)\right),
$$

where each component corresponds to each time period $t=1, \ldots, p$. In this case, the associated average utility, under the additive independence condition, is given by the vector

$$
\left.u_{q}=u\left(S_{q}\right)=\sum_{i=1}^{n} w_{i}^{A} u_{i}^{A}\left(x_{i q}^{A 1}\right), \ldots, \sum_{i=1}^{n} w_{i}^{A} u_{i}^{A}\left(x_{i q}^{A t}\right), \ldots, \sum_{i=1}^{n} w_{i}^{A} u_{i}^{A}\left(x_{i q}^{A p}\right)\right)
$$

and we have now a vector optimisation problem. We have from the vectorial utility a preference relation $\succ_{u}$ on $S$, which leads to a dominance principle defined by

$$
S_{q} \succ_{u} S_{m} \Leftrightarrow u_{q} \geq u_{m},
$$

which means that all the components of $u_{q}$ are greater than or equal to the respective components of $\boldsymbol{u}_{m}$, with at least one strict inequality. The relation $\succ_{u}$ is a strict partial order on $S$, and hence, we state the vector optimisation problem as

$$
\begin{array}{ll}
\max & u\left(S_{q}\right) \\
\text { s.t. } & S_{q} \in S .
\end{array}
$$

A natural concept is that $S_{q} \in S$ is a utility efficient vector alternative if there is no $S_{m} \in S$ such that $\boldsymbol{u}_{m} \geq \boldsymbol{u}_{q}$. This alternative set is called timestream utility efficient vector set and denoted as $E(S, \boldsymbol{u})$. This leads to the problem 'Given $S$ and $\succ_{u}$, find $E(S, u)$ '. Clearly, if $E(S, u)$ had a single element $S_{q}$, it would be the most preferred alternative in the decision making problem. However, this is not the case in most real 
problems, where $E(S, u)$ can contain a lot of elements and is not totally ordered. Thus, our problem is to be restated as 'select a single element from the set $E(S, \boldsymbol{u})$ '. One way to solve this decision problem, favoured by behavioural approaches, will be possible if the DMs are able to reveal more information about their preferences to provide additional structural assumptions and obtain a subset of $E(S, u)$. For this purpose, the DSS includes an interactive metaheuristic, to progressively build an approximation set in collaboration with the DMs, which is valid for any of the settings and based on vectorial optimisation for the utility interval vectors with different dimensions depending on the model used.

This evaluation method, called interactive multiobjective simulated annealing based on an approximation (see [22,23]) is supported by the following idea. The method is based on the set $S$, which is associated with the respective utility vector set $\boldsymbol{u}_{q}$ and involves generating an approximation set $A(S, u)$ of the set $E(S, \boldsymbol{u})$.

The basic idea of multiobjective simulated annealing (MSA) is simple. It begins with a first iteration $S_{0} \in S$, and thus the set $A(S, \boldsymbol{u})$ is initialised containing only $S_{0}$. In the $\mathrm{m}^{\text {th }}$-iteration, another alternative $S_{q}$ from the neighbourhood of the solution in the present iteration, $S_{m}$, is considered, and $S_{q}$ is accepted if it is not dominated by the alternatives currently in the approximation set. In this case, we add $S_{q}$ to the set $A(S, u)$, remove any solution in $A(S, u)$ dominated by $S_{q}$ and do $S_{m+1}=S_{q}$. On the other hand, if $S_{q}$ were dominated by any element in $A(S, \boldsymbol{u})$, we would continue considering $S_{q}$ for the next iteration with a certain probability, known as acceptance probability. In this way, according to the movement in the iterations through the space, we simultaneously build the set $A(S, \boldsymbol{u})$.

The multiobjective rule for the acceptance probability in our context is defined as

$$
\left.\left.P\left(S_{q}, S_{m}, T, \lambda\right)=\min \left\{1, \max _{t} \exp \left\{\lambda_{t} \sum_{i=1}^{n} w_{i}^{A}\left[u_{i}^{A}\left(x_{i q}^{A t}\right)-u_{i}^{A}\left(x_{i m}^{A t}\right)\right]\right) / T\right\}\right)\right\} \text {. }
$$

Some other rules can been defined (see, e.g. [24-26]). $T$ represents the temperature and a weighting vector $\lambda$ is needed to define each function.

Early on (high temperature), almost all moves (that is any updating of the current solution by the randomly selected solution) are accepted. The temperature is then gradually decreased, which means that the algorithm becomes more and more selective about accepting new solutions.

The set $A(S, \boldsymbol{u})$ will depend on $\lambda$, so we shall denote it from now on as $A(S, u, \lambda)$. Thus, $A(S, \boldsymbol{u}, \lambda)$ will contain all the potentially efficient utility solutions generated by MSA using the weighting vector $\lambda$. Note that by controlling the weights we shall be able to increase or decrease the acceptance that might lead us towards a certain region formed by the potential efficient utility solutions.

The procedure for obtaining a good approximation $A(S, u, \lambda)$ of the utility efficient set is as follows: $A(S, \boldsymbol{u}, \lambda)$ is the list of potentially efficient utility solutions obtained with the weights $\lambda$. Taking a weighting vector set $W_{0}$, generated in an extensively diversified way, for each one, MSA will output a list $A(S, u, \lambda)$ that will contain the potentially efficient utility solutions in the direction induced by $\lambda$. The elements of $W_{0}$ are drawn at random from the set $\left\{\lambda \in \Re^{p}: \lambda_{t} \in[0,1] \forall t\right.$ and $\left.\sum_{t=1}^{p} \lambda_{t}=1\right\}$, according to the intra-set point generation proposed in [27], $50 \%$ of which come from the uniform distribution and the remaining from the Weibull 
distribution. Moreover, the weighting vectors $\lambda_{j}, j=1, \ldots, n$, with $\lambda_{j}^{j}=1$ and the remainder equal to 0 , are also included in this set. Thus, to get a good approximation $A(S, \boldsymbol{u})$ for $E(S, \boldsymbol{u})$, we need to filter the set $\bigcup_{\lambda \in W_{0}} A(S, u, \lambda)$ by pairwise comparisons to output the nondominated solutions. This filtering process will be denoted by $\nabla$, in such a way that $A(S, \boldsymbol{u})=\nabla \bigcup_{\lambda \in W_{0}} A(S, \boldsymbol{u}, \lambda)$. Thus, we generate an initial list $L_{0}=\nabla \bigcup_{\lambda \in W_{0}} A(S, \boldsymbol{u}, \lambda)$ as a result of the filtering operation considered above.

In the MSA-based interactive method, a set of minimum satisfaction levels $\varepsilon_{k}$ is used for the different attributes, which represent the bounds within which each one of the utilities of the different components satisfies the DMs individually and limit the input of solutions to the respective solution list.

In the $\mathrm{M}^{\text {th }}$-iteration of the interactive process, the DMs will modify these satisfaction levels according to preferences in response to the information (solution list $L_{M-1}$ ) output in the above iteration. Additionally, the weighting vector set $W_{M-1}$ is updated by discarding the weighting vectors that become useless with respect to the new satisfaction levels. Finally, MSA is run taking each element in the updated weighting vector set and the filtering process is performed taking into account the solution list in the above iteration as well, which leads to list $L_{M}$.

$$
L_{M}=\nabla\left\{L_{M-1} \cup\left\{A(S, u, \lambda), \lambda \in W_{M}\right\}\right\} \cap\left\{S_{q}: \sum_{i=1}^{n} w_{i}^{A} u_{i}^{A}\left(x_{i q}^{A t}\right) \geq \varepsilon_{t}, \forall t\right\} .
$$

Interactive MSA ends when the DMs are completely satisfied with a solution or set of solutions of $L_{M}$.

Figure 6 shows a display of the minimum satisfaction levels and MSA parameters that the DMs must provide to try to reach a final solution interactively.

Figure 6 Visualising the minimum satisfaction levels and multiobjective simulated annealing parameters that lead to the final solution

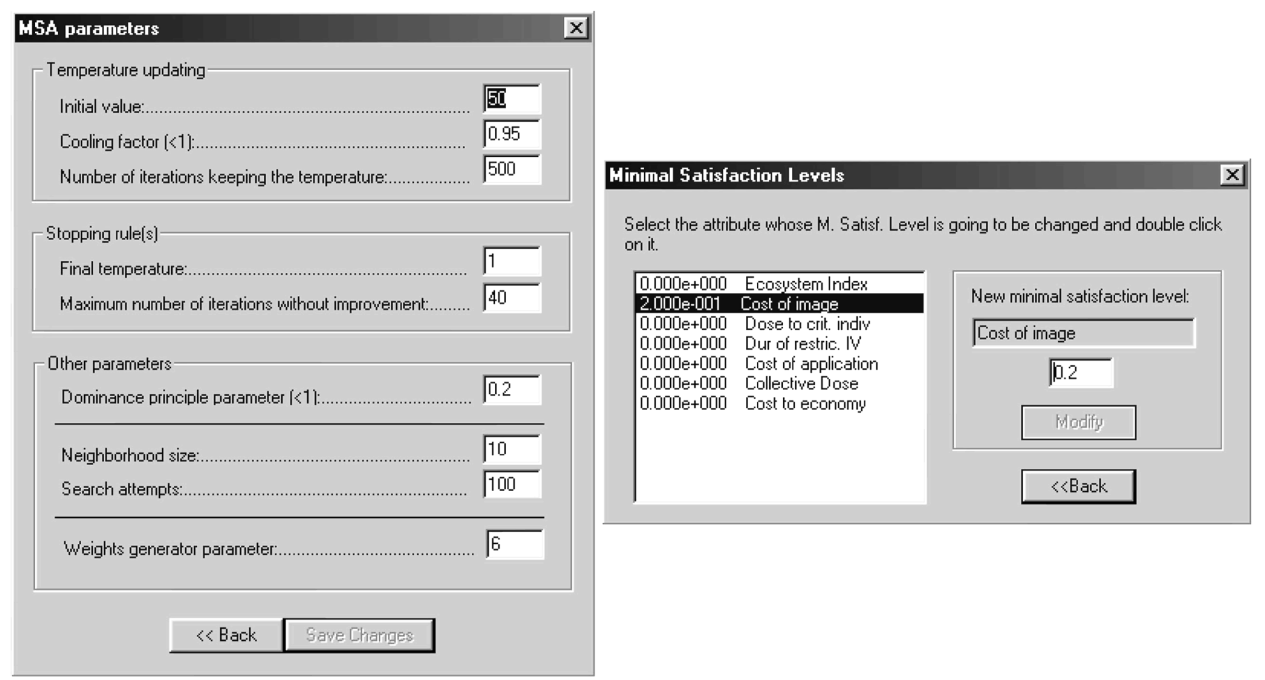


The role that many of the parameters in Figure 6 play in interactive simulated annealing is defined in $[23,28]$.

\subsection{A dynamic case with imprecision. The discounting approach}

How to trade off the present and the future is a difficult question, and the classical economic answer is to use exponential discounting. There has been lengthy discussion on this important point, where the prevailing tendency in normative economic theory has been to demand a constant discount rate, although descriptive research shows that few people value the future in this way. On the other hand, psychological research has been carried out to investigate how people discount various commodities over time and what affects their discount rate. One conclusion is that cognitive theories claim that this matter is because people fail to picture the future adequately and are averse to waiting. In [29] the issues inherent in discounting non-market goods are discussed, arguing that it is unreasonable to do so. Thus, we could say that the research shows that discount rates depend on the decision context and framing. The stationary axiom of discounted utility theory, see [18], states that the preference over two outcomes is independent of the time when the outcomes are evaluated. Therefore, the preference should depend only on the absolute time difference between outcomes. But some authors claim that this does not agree with exhibited value judgements, and there are persuasive reasons why it should. Hyperbolic discount rates (see [30,31]) do allow DMs to attach more importance to the very long-term future than constant discount rates. This is something that should be explored.

To illustrate the consideration of discount rates, we finally show the most involved case, MODEL 16, a dynamic model with imprecision concerning all the inputs. For each alternative $S_{q} \in S$, the consequence is an outcome stream

$$
\begin{aligned}
& \left(\left(\left\lfloor x_{1 q}^{L 1}, x_{1 q}^{U 1}\right\rfloor, \ldots,\left\lfloor x_{n q}^{L 1}, x_{n q}^{U 1}\right\rfloor\right), \ldots,\left(\left\lfloor x_{1 q}^{L t}, x_{1 q}^{U t}\right\rfloor, \ldots,\left\lfloor x_{n q}^{L t}, x_{n q}^{U t}\right\rfloor\right), \ldots,\right. \\
& \left.\left(\left\lfloor x_{1 q}^{L p}, x_{1 q}^{U p}\right\rfloor, \ldots,\left\lfloor x_{n q}^{L p}, x_{n q}^{U p}\right\rfloor\right)\right) .
\end{aligned}
$$

Without discount factors the utility of alternative $S_{q}$ is, under the additive independence condition and for increasing utility functions,

$$
\begin{aligned}
\boldsymbol{u}_{q}=\boldsymbol{u}\left(S_{q}\right)= & \left(\left[\sum_{i=1}^{n} w_{i}^{L} u_{i}^{L}\left(x_{i q}^{L 1}\right), \sum_{i=1}^{n} w_{i}^{U} u_{i}^{U}\left(x_{i q}^{U 1}\right)\right], \ldots,\right. \\
& {\left.\left[\sum_{i=1}^{n} w_{i}^{L} u_{i}^{L}\left(x_{i q}^{L t}\right), \sum_{i=1}^{n} w_{i}^{U} u_{i}^{U}\left(x_{i q}^{U t}\right)\right]\right) }
\end{aligned}
$$

and a possible solution is obtained by applying the above-mentioned interactive simulated annealing. We shall have the same natural concept as above, i.e. an alternative $S_{q} \in S$ is an imprecise utility efficient vector alternative if there is no $S_{m} \in S$ such that $\boldsymbol{u}_{m} \geq \boldsymbol{u}_{q}$. This set of alternatives will now be called imprecise timestream utility efficient vector set and denoted by $E_{\mathrm{I}}(S, u)$, where subscript I means imprecision. The problem-solving procedure based on interactive simulated annealing will also be appropriate here. 
With discount factors, given the stationarity condition combined with additive independence, the utility vector for the alternative $S_{q}$ is

$$
\boldsymbol{u}_{q}=\boldsymbol{u}\left(S_{q}\right)=\left[\sum_{t=1}^{p} \sum_{i=1}^{n} \rho_{i}^{t-1} w_{i}^{L} u_{i}^{L}\left(x_{i q}^{L t}\right), \sum_{t=1}^{p} \sum_{i=1}^{n} \rho_{i}^{t-1} w_{i}^{U} u_{i}^{U}\left(x_{i q}^{U t}\right)\right]
$$

where the first component corresponds to the lower endpoints of the imprecise consequences and the second component to the upper ones, for some discount factors $\rho_{i}$. Figure 7 shows a display of the utility intervals $u_{q}$ (including the average values) for any available alternatives after applying the discount factor.

Figure 7 The utility intervals and the ranked alternatives based on their precise utilities

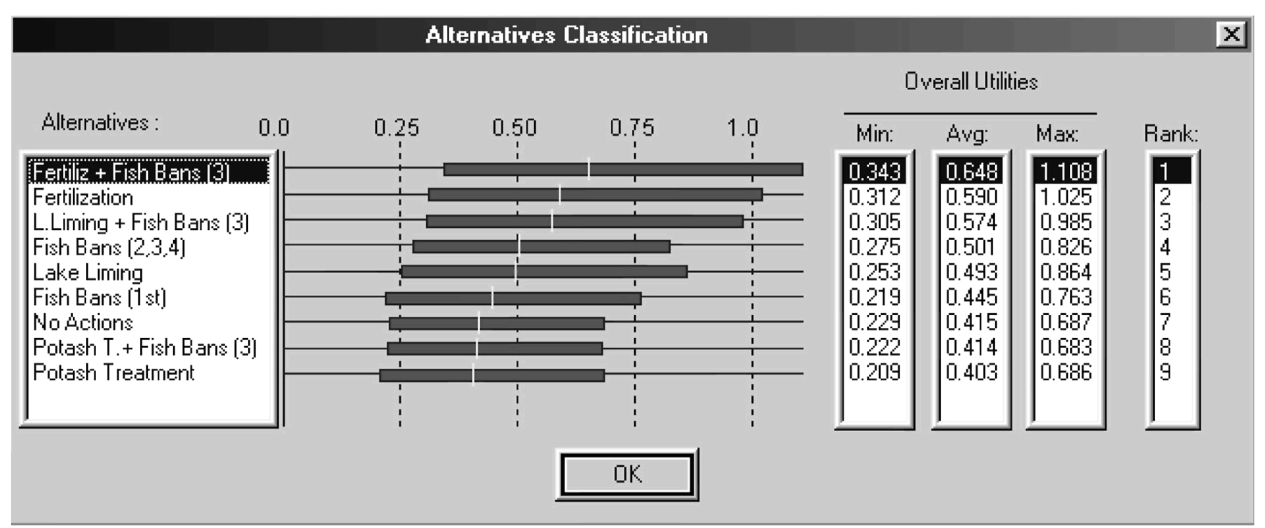

As over medium to long time spans, i.e. for large $p, \rho_{i}^{t-1}$ becomes very small as $t$ tends to $p$, which means that consequences towards the end of the timestream are effectively discounted to zero. We have also implemented an approach proposed in [31,32], which suggests a varying discount rate that could devaluate the future at a much slower rate. If the DM is timing averse, the utility of a future stream of multiattributed outcomes is

$$
\begin{aligned}
& {\left[w_{1}^{L} u_{1}^{L}\left(x_{1 q}^{L t}\right)+\sum_{i=2}^{n} \sum_{t=1}^{p}\left(\frac{b}{b+t}\right)^{r} w_{i}^{L} u_{i}^{L}\left(x_{i q}^{L t}\right),\right.} \\
& \left.w_{1} u_{1}^{U}\left(x_{1 q}^{U t}\right)+\sum_{i=2}^{n} \sum_{t=1}^{p}\left(\frac{b}{b+t}\right)^{r} w_{i}^{U} u_{i}^{U}\left(x_{i q}^{U t}\right)\right]
\end{aligned}
$$

where $b>0$ is the temporal mid-value, that is, the point in the future given half of the present weight, and $-\infty<r<\infty$ reflects how the ratio of the outcomes is related to the ratio of the time effects. Note that the values in the above expression decay to 0 , as time tends to infinity slower than in the previous one and, therefore, this approach attaches relatively more importance to the long-term outcomes. The model looks at the ratio between two time periods and not at the absolute difference in their time.

To use this last model, it is necessary to determine $b$ and $r$ for the DMs. If the DMs give an initial discount rate $d$, then $b=1 / d$. Alternatively, the DMs can be asked to provide their temporal mid-value. Assuming that the present is given a value 
1 , then the temporal mid-value will be given a value 0.5 and this point in time is the value of $b$. Harvey (see [32]) advises asking DMs for their temporal mid-value to determine $b$, as this encourages them to think about the whole project lifetime and determine the middle of that range with respect to importance. The factor $r$ is determined by analysing how the ratio of the outcomes is related to the ratio of the time tradeoffs, see [32].

The consideration of the discount approach for MODEL 9, with different discount factors $\rho_{i}$, one for each attribute, provides the utility

$$
u\left(S_{q}\right)=\sum_{t=1}^{p} \sum_{i=1}^{n} \rho_{i}^{t-1} w_{i}^{A} u_{i}^{A}\left(x_{i q}^{A t}\right)
$$

leading to a scalar instead of a vector optimisation problem. Also, considering Harvey's approach for this model, then the utility of a future stream of multiattributed outcomes, which takes into account the DMs' time aversion, is given by

$$
u\left(S_{q}\right)=w_{1}^{A} u_{1}^{A}\left(x_{1 q}^{A 1}\right)+\sum_{i=2}^{n} \sum_{t=1}^{p}\left(\frac{b}{b+t}\right)^{r} w_{i}^{A} u_{i}^{A}\left(x_{i q}^{A t}\right)
$$

where $b>0$ is the temporal mid-value and $-\infty<r<\infty$ reflects how the ratio of the outcomes is related to the ratio of the time effects.

In short, we have that each alternative $S_{q}$ will be characterised in all the above cases by a vector of utility intervals

$$
u\left(S_{q}\right)=u_{q} \equiv\left(\left\lfloor u_{q}^{L 1}, u_{q}^{U 1}\right\rfloor \ldots,\left\lfloor u_{q}^{L p}, u_{q}^{U p}\right\rfloor\right)
$$

of dimension from 1 to $p$, depending on the model adopted, which is solved by the DSS using interactive simulated annealing in the vectorial case.

Finally, we note that the utility and weight intervals together with the value intervals are used in sensitivity analysis to gain insight and confidence in the ranking of the alternatives, and as an aid for reducing, if possible, the alternatives set.

\section{Sensitivity analysis}

Sensitivity analysis (SA), which essentially involves examining changes in the ranking as a function of the input parameters (weights, utilities and consequences) varying within a reasonable range, can give further insight into the robustness of the recommendations. Some types of sensitivity analysis are described in [19], which introduces a framework for sensitivity analysis in multiobjective decision making. SA is usually performed by changing the weights or utilities and observing their impact on the ranking of alternatives. Hence, if the DMs make a change to a bound or average weight, the DSS takes cares of how these changes are propagated through the objective hierarchy and automatically recalculates the overall utility for each alternative and the resulting ranking. 
Another way of performing SA involves assessing the interval within which a weight can vary, maintaining a constant ratio among the other weights without affecting the overall alternative ranking. Suppose that we currently have a ranking of the given alternatives and the DMs choose a node or leaf of the objective tree with an associated weight. The system calculates the weight interval for this node/leaf, taking into account the updated weights for the objectives stemming from its predecessor, so that the ranking does not change. In other words, if the weight is changed and the new value is within the range, then the ranking will not change. However, if the new value is not within the range, the new ranking will be different from the previous one. Figure 8 shows a display of a weight stability interval for a certain objective.

Figure 8 Weight stability intervals for an objective

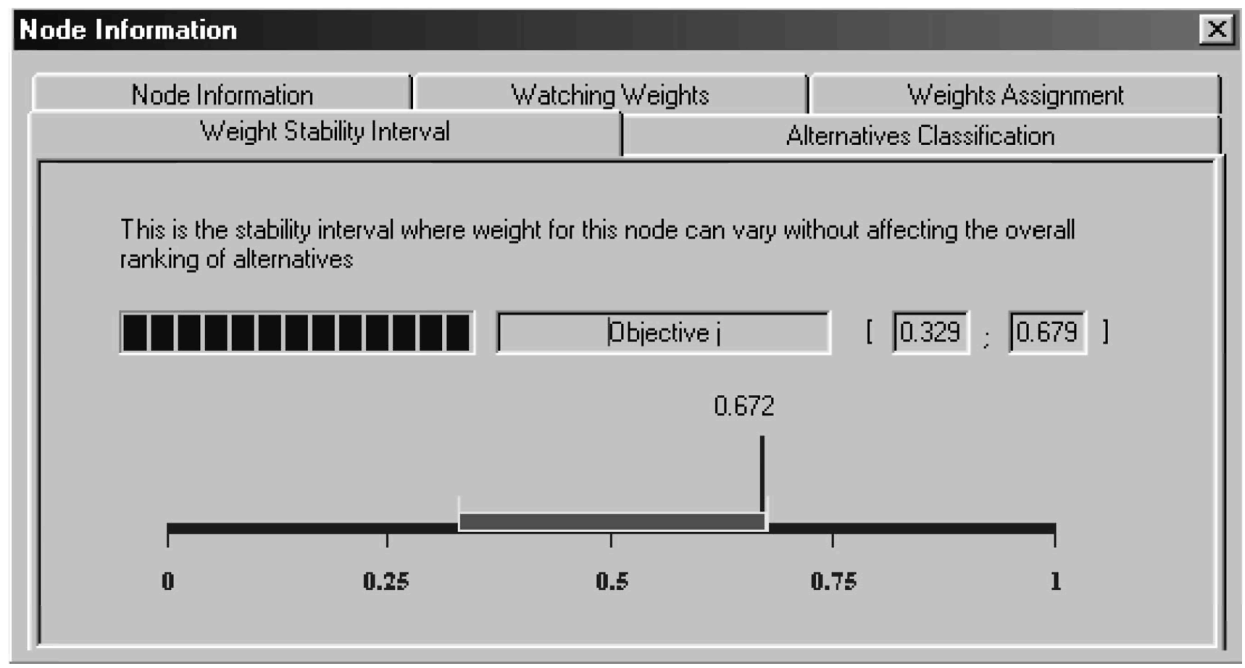

Finally, the system runs simulation techniques for SA. This kind of sensitivity analysis allows for simultaneous changes in weights and generates results that can be easily analysed statistically to provide more insights into the multiattribute model recommendations. We propose selecting weights at random using a computer simulation programme so that the results of many combinations of weights, including a complete ranking, can be explored efficiently (see [19]).

Three general classes of simulation are implemented: random weights, rank order weights and response distribution weights. They are briefly described below. 
1 Random weights: As an extreme case, attribute weights are generated completely at random. This approach implies no knowledge whatsoever of the relative importance of attributes. In many multicriteria settings, the scores of the alternatives significantly limit the subset of potential rankings.

2 Rank order weights: Randomly generating weights while preserving an attribute rank order places substantial restrictions on the domain of possible weights that are consistent with the DMs' judgement of attribute importance. Therefore, the results from the rank order simulation may provide more meaningful results. The DMs can introduce the rank order for all or only some of the attributes of the problem.

3 Response distribution weights: The third type of simulation-based sensitivity analysis recognises that the weight assessment procedure is subject to variation. For a single DM, this variation may be in the form of response error associated with weight assessment. Thus, whereas in the first class of simulation, attribute weights were randomly assigned values between 0 and 1 (taking into account that the sum of the whole is 1), attribute weights are now randomly assigned values taking into account the attribute normalised weight intervals provided by the DM through the weights assignment methods.

Once the simulation has been run, the DSS computes several statistics about the rankings of each alternative, like mode, minimum, maximum, mean, standard deviation and the 25 th, 50 th and 75 th percentiles. This information can be useful for discarding some possible alternatives, aided by a display which presents a multiple boxplot for the alternatives, see Figure 9 .

Figure 9 Vertically grouped boxplot for the results of the simulation of eight alternatives considering random weights

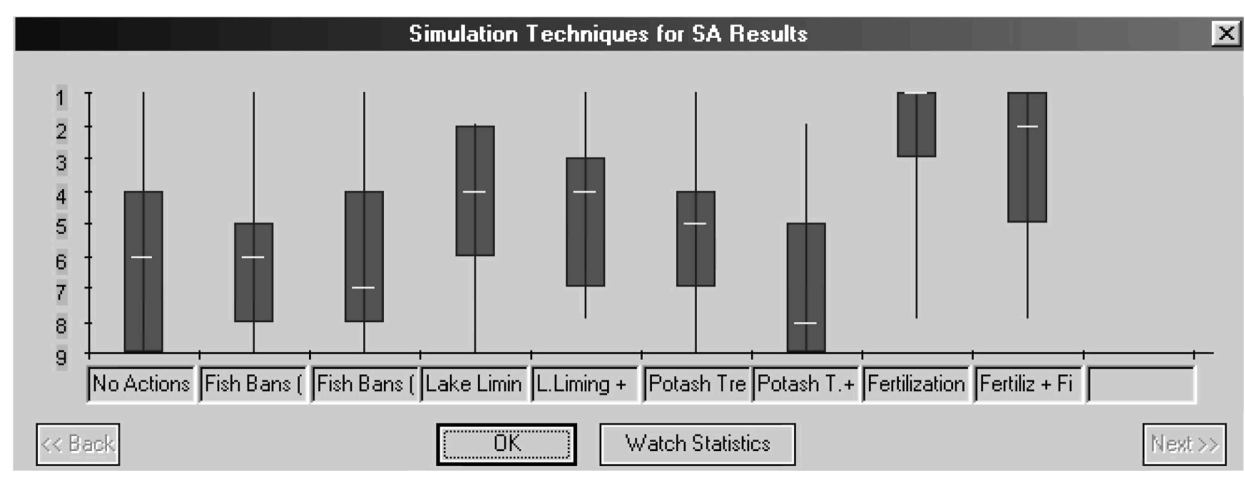

Thus, the DSS includes a multiparametric sensitivity analysis facility to gain additional insight into the alternative ranking, by means of which it can be demonstrated that these optimisation problems will be more tractable using the temporal domain to take into account their effects. 


\section{Summary}

We have built a DSS for the Decision Analysis process based on imprecise inputs concerning utilities, weights and consequences and supported by a dynamic objective tree structure, which is intended to provide the grounds for the description and evaluation of hypothetical management alternatives or decision alternatives for the different scenarios to be considered. The relevant objectives, as well as the attributes associated with the lowest-level objectives of the hierarchy, are created in the DSS, the idea being that the scenarios need to contain enough technical information for them to be realistic, while not being so technical as not to be understandable. These models must be analysed with respect to the attributes associated with the lowest-level objectives of the hierarchy, some of which (may be all) are time dependent. Next, the DMs will have to assess the imprecise utility functions, scaling constants and the discount factors, if deemed appropriate, which will be used to construct the considered models, associating each alternative with a vector of utility intervals. The application of interactive simulated annealing for dimension 2 or greater will lead to the determination of the best or a satisfactory alternative.

\section{Acknowledgements}

This paper was supported by the Spanish Ministry of Science and Technology project DPI2001-3731 and the Madrid regional government project CAM 07T/0027/2000.

\section{References}

1 Von Neumann, J. and Morgenstern, O. (1947) Theory of Games and Economic Behaviour, Princeton University Press.

2 Savage, L.J. (1954) The Foundations of Statistics, New York: John Wiley.

3 Fishburn, P.C. (1970) Utility Theory for Decision Making, New York: John Wiley.

4 Mateos, A., Ríos-Insua, S. and Gallego, E. (2001) 'Postoptimal analysis in a multi-attribute decision model for restoring contaminated aquatic ecosystems', Journal of the Operational Research Society, Vol. 52, pp.1-12.

5 Brownlow, S.A. and Watson, S.R. (1987) 'Structuring multi-attribute value hierarchies', Journal of the Operational Research Society, Vol. 38, pp.309-317.

6 Keeney, R.L. and Raiffa, H. (1993) Decision with Multiple Objectives. Preferences and Value Tradeoffs, Cambridge University Press.

7 Gallego, E., Ríos-Insua, S., Mateos, A. and Ríos Insua, D. (1998) 'Contaminated aquatic ecosystems restoration: a case study', in F.J. Girón (Ed.) Applied Decision Analysis, Boston, USA: Kluwer, pp.19-34.

8 Ríos Insua, D., Gallego, E., Mateos, A. and Ríos-Insua, S. (2000) 'MOIRA: a decision support system for decision making on aquatic ecosystems contaminated by radioactive fallout', Annals of Operations Research, Vol. 95, pp.341-364.

9 Raiffa, H. (1970) Decision Analysis. Introductory Lectures on Choices under Uncertainty, Reading, Mass, USA: Addison-Wesley Publishing Company. 
10 Goodwin, P. and Wright, G. (1998) Decision Analysis for Management Judgment, New York: John Wiley.

11 Clemen, R.T. and Reilly, T. (2001) Making Hard Decisions with Decisions Tools, Australia: Duxbury, Thomson Learning.

12 Weber, M. (1987) 'Decision making with incomplete information', European Journal of Operational Research, Vol. 28, pp.44-57.

13 Hershey, J.C., Kunreuther, H.C. and Schoemaker, P.J. (1982) 'Sources of bias in assessments procedures for utility functions', Management Science, Vol. 28, pp.936-953.

14 Jaffray, J.Y. (1989) 'Some experimental findings on decision making under risk and their implications', European Journal of Operational Research, Vol. 38, pp.301-306.

15 Farquhar, P.H. (1984) 'Utility assessment methods', Management Science, Vol. 30, pp. $1283-1300$.

16 von Nitzsch, R. and Weber, M. (1988) 'Utility function assessment on a micro-computer: An interactive procedure', Annals of Operations Research, Vol. 16, pp.149-160.

17 Ríos, S., Ríos-Insua, S., Ríos Insua, D. and Pachon, J.G. (1994) 'Experiments in robust decision making', in S. Ríos (Ed.) Decision Theory and Decision Analysis: Trends and Challenges, Boston: Kluwer, pp.233-242.

18 French, S. (1986) Decision Theory: An Introduction to the Mathematics of Rationality, Ellis Horwood.

19 Jiménez, A., Ríos-Insua, S. and Mateos, A. (2002) 'A decision support system for multiattribute utility evaluation based on imprecise assignments', Decision Support Systems, Vol. 36, pp.65-79.

20 Raiffa, H. (1982) The Art and Science of Negotiation, Cambridge, Mass.: Harvard University Press.

21 Stewart, Th.J. (1996) 'Robustness of additive value function method in MCDM', Journal of Multi-Criteria Decision Analysis, Vol. 5, pp.301-309.

22 Ríos-Insua, S., Jiménez, A. and Mateos, A. (2001) 'Solving imprecise discrete multiattribute problems under risk based on interactive simulated annealing', in K.J. Engemann and G.E. Lasker (Eds.) Advances in Decision Technology and Intelligent Information Systems, Ontario, Canada: The International Institute for Advances Studies in Systems Research and Cybernetics, pp.54-58.

23 Jiménez, A., Ríos-Insua, S. and Mateos, A. (2002) 'Interactive simulated annealing for solving imprecise discrete multiattribute problems under risk', Pesquisa Operacional, Special Issue on Data Envelopment Analysis and Multicriteria Decision Aid, Vol. 22, pp.265-278.

24 Serafini, P. (1992) 'Simulated annealing for multiple objective optimization problems', G.H. Tzeng, H.F. Wang, V.P. Wen and P.L. Yu (Eds.) Proceedings of the Tenth International Conference on MCDM, Berlin: Springer Verlag, pp.87-96.

25 Fortemps, P., Teghem, J. and Ulungu, B. (1994) 'Heuristics for multiobjective combinatorial optimization by simulated annealing', XIth International Conference on $M C D M$, Coimbra, Portugal.

26 Kubonati, H. and Yoshimura, K. (2003) 'Performance evaluation of acceptance probability functions for multi-objective sensitivity analysis', Computers and Operations Research, Vol. 30, pp.427-442.

27 Steuer, R.E. and Harris, F.W. (1980) 'Intra-set point generation and filtering in decision and criterion space', Computers and Operations Research, Vol. 7, pp.45-53.

28 Jiménez, A. (2002) 'Un Sistema de Ayuda a la Decisión Multiatributo con Asignaciones Imprecisas', PhD Thesis, Madrid Technical University.

29 Atherton, E. and French, S. (1997) 'Issues in supporting intertemporal choice', in M. Karwan, J. Spronk and J. Wallenius (Eds.) Essays in Decision Making, Berlin: Springer-Verlag. 
30 Ahlbrecht, M. and Weber, M. (1995) 'Hyperbolic discount models in prescriptive theories of intertemporal choice', Zeitschrift fur Wirtschafts, Vol. 115, pp.535-568.

31 Harvey, C. (1992) 'A slow discounting model for energy conservation', Interfaces, Vol. 22, pp. $47-60$.

32 Harvey, C. (1995) 'Proportional discounting of future benefits and costs', Mathematics of Operations Research, Vol. 20, pp.381-399. 